\title{
Gubernamentalidad neoliberal y producción de conocimiento en la universidad: genealogía de una configuración subjetiva*
}

\author{
Neoliberal Governmentality and Knowledge Production in \\ Higher Education: Genealogy of a Subjective Configuration
}

Recibido: 4 de noviembre de 2014 | Aceptado: 1 de octubre de 2015

\author{
LUCÍA GÓMEZ SÁNCHEZ ** \\ FRANCISCO JÓDAR RICO **** \\ MARÍA Jesús BRAVO SÁNCHEZ \\ Universitat de València, España
}

doi :10.11144/Javeriana.upsy14-5.gnpc

Para citar este artículo: Gómez, L., Bravo, M. J., \& Jódar, F. (2015). Gubernamentalidad neoliberal y producción de conocimiento en la universidad: genealogía de una configuración subjetiva. Universitas Psychologica, 14(5), 1735-1750. http://dx.doi. org10.11144/Javeriana.upsy14-5.gnpc

* Artículo de investigación, sin financiación.

** Facultad de Psicología, Departamento de Psicología Social. Avda. Blasco Ibáñez 21, 46010 Valencia (España).Correos electrónicos: gomezs@uv.es; maria.j.bravo@uv.es

**** Departamento de Didáctica y Organización Escolar. Correo electrónico: francisco.jodar@uv.es

\section{RESUMEN}

En este artículo, se parte de la consideración de que cualquier interrogación sobre las condiciones de posibilidad de una investigación crítica debe hacerse teniendo en cuenta el proceso de mercantilización del conocimiento, impulsado por la incorporación de los modos de gubernamentalidad neoliberal en la universidad. Proceso este que redefine el valor y el uso que se le da al conocimiento producido y redefine también las subjetividades de los colectivos universitarios: formas de entender el conocimiento y configuraciones subjetivas que promueven modos instrumentales de relacionarse con el conocimiento en un escenario individualizador y competitivo. Por ello, se considera políticamente relevante llevar a cabo un análisis genealógico de esta forma singular de relación con el conocimiento, que muestre su carácter contingente señalando los discursos y prácticas que lo configuran y sostienen. Esta genealogía permitirá explorar las sujeciones que imposibilitan una producción de conocimientos críticos, así como desnaturalizar su carácter de evidencia.

Palabras clave

neoliberalismo; subjetividad; gubernamentalidad; genealogía; conocimiento; crítica A B S T R A C T

This article claims that any questioning of the conditions related to the feasibility of critical research projects needs to be done by taking into account the process of commodification of knowledge that has developed in universities due to the incorporation of the modes of neoliberal governmentality. This is a process which redefines the value and use that we give to produced knowledge as well as the subjectivity of university collectives. The way knowledge is understood and the subjective configurations that promote instrumental ways of relating to knowledge in an individualizing and competitive environment need to be taken into account. Therefore, we consider it politically relevant to carry out a genealogical analysis of this singular way to treat knowledge in order to show its conceivable traits by pointing out the discourses and practices that shape and support this perspective. This genealogy will enable us to explore what makes the production of critical knowledge impossible and distorts its proof-based character when developed in such an environment.

Keywords

neoliberalism; subjectivity; governmentality; genealogy; knowledge; criticism 


\section{Un plano de interrogación: crítica, subjetividad y política del conocimiento}

Interrogarnos, de forma situada, sobre aquello que definiría el pensamiento crítico requiere prestar atención, como tarea previa, a las coordenadas que enmarcan hoy la tarea investigadora y que responden a la incorporación de políticas neoliberales en la universidad. Dichas políticas constituyen formas de gubernamentalidad que han modificado tanto el valor y el uso que le damos al conocimiento como la subjetividad de los colectivos universitarios (Amigot \& Martínez 2013, 2015; Gómez \& Jódar, 2013; Montenegro $\&$ Pujol, 2010) ${ }^{1}$. Estas formas de conocimiento y formas de subjetividad, que se sostienen en una lógica mercantilizada e individualizada y que han conseguido imponerse como un hecho dado, resultan incontestables, evidentes, neutrales, constituyendo una realidad frente a la cual lo único que se puede hacer es adaptarse (Laval \& Dardot, 2010/2013).

En este escenario, consideramos que la dificultad para desarrollar una investigación crítica no radica únicamente en el ámbito de los sentidos (temáticas, perspectivas, problemas) hacia los que orientamos nuestras investigaciones sino en el tipo de relación que mantenemos con el conocimiento y con nosotros mismos. Por ello, creemos necesario prestar atención a nuestra política del conocimiento, a las condiciones en las que este debe ser producido, a los requerimientos a los que debe responder, a la forma que debe adoptar para tener valor, a la propia definición de valor, a los

1 El neoliberalismo entendido como modo de gubernamentalidad no hace referencia únicamente a un modelo socioeconómico u organizacional, sino a una transformación de la subjetividad, mediante procedimientos prácticos o tecnologías, entendidas como agenciamientos múltiples y heterogéneos de discursos y prácticas, que pretenden conformar, normalizar, guiar, instrumentalizar, modelar las ambiciones, aspiraciones, pensamientos y acciones de los sujetos, para lograr los fines que se consideran deseables. Desde la perspectiva de la gubernamentalidad, que permite entender el carácter político de cualquier configuración identitaria, somos el resultado de tecnologías de gobierno que toman modos de ser humanos como objeto (Barry, Osborne, \& Rose, 1996; Burchell, 1996; Burchell, Gordon, \& Miller, 1991; Dean, 1999; Foucault, 1978a, 1978b, 1981; Rose, 1996a, 1996b, 1999; Rose \& Miller, 1992). medios en los que se difunde y a los efectos que genera su difusión (Foucault, 1977), pero hacerlo desde un ángulo que atienda a la particular configuración subjetiva que esta forma de entender el conocimiento requiere y genera.

Asumimos que la producción de contenidos críticos es difícilmente compatible con enfoques epistemológicos y metodológicos cientificistas y tecnocráticos que naturalizan la realidad social (Ema, 2013). Reconocemos el valor de aquellos análisis que analizan las formas de dominación y los procesos de hegemonización presentes, en lugar de consolidarlas (Boltanski, 2014; Ibáñez, 1989). Y constatamos que la pluralidad temática y de perspectivas propia de los saberes de las ciencias sociales y humanas queda neutralizada por la estandarización de los procedimientos de investigación y difusión, produciendo investigaciones disciplinadas incompatibles con el pensamiento crítico (Garcés, 2013). Sin embargo, queremos poner el foco en la configuración subjetiva que producen los modos de gobierno neoliberales, la del investigador empresario de sí mismo (Rose, 1996b), porque sostiene (y se sostiene), como mostraremos a lo largo del trabajo, en una concepción privatizada del conocimiento que pone en peligro su dimensión social. Y ello, en dos sentidos vinculados intrínsecamente tanto a lo que entendemos por investigación crítica como a la misma función de la universidad pública: la necesaria implicación y conexión del conocimiento con realidades y problemas con el objetivo de colaborar en procesos de transformación social, por un lado y su valor como bien común que debe circular para producir efectos, alianzas y resonancias, por otro.

El horizonte normativo que permite señalar estas dos funciones críticas del conocimiento y de la institución universitaria no responde a un principio abstracto, sino que emerge directamente de distintas experiencias surgidas en los últimos años (universidades experimentales, editoriales, librerías, centros sociales...) que cuestionan la forma hegemónica de entender la producción de conocimiento en el espacio universitario precisamente por su incapacidad para generar pensamiento 
y prácticas críticas ${ }^{2}$. Movimientos, colectivos, posiciones preocupadas por generar saberes con capacidad antagonista y que ponen en un primer plano cuestiones que el saber académico invisibiliza, permitiendo materializar otra relación con el conocimiento: ipara qué investigamos?, ipara quiénes investigamos?, icon quiénes pensamos?, ¿qué efectos perseguimos?

La propia investigación que queremos llevar a cabo y nuestra posición subjetiva está atravesada por la reapropiación neoliberal del conocimiento y participa de las contradicciones y paradojas a las que apuntamos: iSe puede producir un saber crítico cuando la misma producción de conocimiento se ha convertido en un modo de gobierno neoliberal de la subjetividad?, ¿Cuándo la subjetividad de quien produce conocimiento es funcional a las formas de dominación que pretende subvertir? Por ello, el modo que tenemos de entender la tarea crítica se vincula con el esfuerzo de analizar genealógicamente un modo de subjetividad que es el nuestro, de interrogarnos por los límites (políticos) que determinan nuestras conductas, deseos y aspiraciones, por la forma, en definitiva, en que estamos siendo gobernados. Intentando, de ese modo, una desujeción en una política del conocimiento que nos configura, pero que cuestionamos.

De este modo, el recorrido de este trabajo será el siguiente: en primer lugar, describiremos brevemente cómo la producción de conocimiento en la universidad entra en un circuito de mercado que exige incrementar la producción investigadora y encauzarla de acuerdo a criterios de calidad que permitan competir, mostrando la configuración

2 Estos espacios constituyen laboratorios de autoformación que pretenden construir formas de pensamiento que tengan capacidad real de incidir, de producir efectos en lo social. Conforman instituciones del común situadas al margen de la institución universitaria y de sus canales de difusión del conocimiento, apostando por la cultura libre y por prácticas colectivas de producción de saberes. En el Estado español incluyen colectivos o grupos como Universidad Nómada, Universidad Libre Experimental, Observatorio Metropolitano); editoriales (Traficantes de Sueños), proyectos (Nociones Comunes, Manos Invisibles), librerías y centros sociales (Ateneu Candela, Casa Invisible) muchos de ellos agrupados en la Fundación de los Comunes. Sobre el alcance político de estas experiencias véase Herreros (2010) y Garcés (2010). subjetiva que requieren estas lógicas mercantilizadoras: investigadores que adoptan criterios empresariales como forma reflexiva. A continuación, y en segundo lugar, llevaremos a cabo una genealogía de esa forma individualizada de relacionarnos con el conocimiento, mostrando los discursos y prácticas que la han configurado y que la sostienen así como sus implicaciones a la hora de llevar a cabo investigaciones críticas. Una genealogía que visibilice los elementos más potentes que los modos de gobierno neoliberal ponen en juego con el objetivo de contribuir a desnaturalizar su carácter de evidencia. Y, en tercer lugar, en las reflexiones finales, exploraremos las sujeciones que imposibilitan una producción de conocimientos críticos apuntando también, de forma tentativa, a propuestas de nuevas prácticas institucionales.

\section{Sujetos e investigaciones en un circuito de mercado}

La progresiva incorporación de discursos y prácticas neoliberales en la universidad española vinculados, en los últimos años, al proceso de construcción del Espacio Europeo de Educación Superior y del Espacio Europeo de Investigación ha instaurado la competitividad como lógica organizadora de la producción de conocimiento. En un contexto de reducción del gasto público, en Educación Superior y de financiación competitiva y diferenciada que cuestiona cualquier protección asegurada, se insta a las universidades a responsabilizarse de su sostenibilidad financiera con criterios de productividad donde la investigación tiene un papel central (Comisión Europea, 2003, 2005, 2006). Esto se traduce en dos exigencias intrínsecamente relacionadas. Por un lado, la exigencia de competir a escala estatal, europea y global por recursos, contratos, tasas de matrícula, fondos para investigación y para el propio desarrollo institucional, alianzas con empresas privadas a través de la consecución de una posición destacada en las jerarquías o rankings. Y por otro, la exigencia continua y siempre inacabada de evaluación y rendición de cuentas a la que deben someterse los colectivos universitarios para garantizar la propia empleabilidad y desarrollo profesional. 
La exigencia de competir explica la necesidad de aumentar la producción investigadora y de hacerlo encauzando, disciplinando la producción de conocimiento de acuerdo a criterios globales de productividad/calidad que permiten competir en el mercado de la educación superior. De este modo, en este espacio de capitalismo académico (Slaughter \& Leslie, 1997) el conocimiento se somete progresivamente a regulaciones, clasificaciones y jerarquías donde priman los indicadores de producción que tienen valor en circuitos legitimados: "el conocimiento es un capital pero es necesario identificar en él lo que realmente tiene de valor para el mercado" (Ministerio de Ciencia e Innovación, 2008, p. 38). Se valora lo reducido a mensurable, los productos susceptibles de ser cuantificados y exhibidos (Laval \& Dardot, 2010/2013) 3 .

La calidad investigadora se objetiva formalmente para permitir su medición y la competición misma. Criterios de valor centrados no en procesos intrínsecos -al margen de las valoraciones que se realizan en determinadas entidades con carácter interno-, sino extrínsecos; en la calidad de la publicación en que se difunde, avalada por el cumplimiento de determinados estándares formales, pero sobre todo por el número de citas que acumula ${ }^{4}$. La investigación así definida y evaluada prescribe la forma que debe tomar la producción de conocimiento.

La visibilidad y la posición de la universidad depende de los productos que se derivan de la actividad investigadora, por ello, se asume que para conseguir estos objetivos institucionales es necesario promover cambios de cultura integrales y en los modelos de trabajo que favorezcan la implicación de todos los colectivos universitarios, poniendo en

3 Los indicadores de producción/productividad sometidos a evaluación son fundamentalmente: artículos en revistas indexadas en el JCR, tramos de investigación reconocidos (sexenios), proyectos $\mathrm{I}+\mathrm{D}$, tesis doctorales, becas FPU, doctorados con mención hacia la excelencia y patentes.

4 La publicación de artículos en determinadas revistas científicas (aquellas que se incluyen en determinadas bases de datos internacionales con índices medibles de impacto como Web of Science [WOS] del Journal Citation Report) se considera el criterio que valida el conocimiento producido, en detrimento de otros formatos y modos de canalizar la actividad investigadora. juego de manera productiva sus capacidades (Comisión Europea, 2005, 2006, 2008; Ministerio de Ciencia e Innovación, 2008, 2010). La movilización "del fondo común de conocimientos, talento y energía existente en las universidades" (Comisión Europea, 2006, p. 5) requiere procesos de reconfiguración identitaria. Se trata de favorecer alianzas entre las necesidades de la organización y las necesidades subjetivas, hace converger prioridades institucionales con los deseos individuales (Rose, 1989, 1996a).

Desde el punto de vista de la redefinición subjetiva, consideramos que el proceso de reforma neoliberal en curso está siendo un éxito. El proceso que estamos describiendo donde la producción de conocimiento entra en un circuito de mercado ha sido capaz de construir su propio sujeto, de modelar investigadores emprendedores dispuestos a comprometerse en el proceso permanente de competencia por contratos, publicaciones, proyectos (Ball \& Youdell, 2007). Con ello, no negamos la pluralidad que afecta a cada profesor/a, atravesado por discursos diferentes o por discursos que no resultan armónicos con las prácticas que realizan o que se les imponen. Es decir, este proceso de redefinición identitaria no se manifiesta de forma monolítica y homogénea, sino de forma fragmentada, heterogénea y paradójica. Sin embargo, a pesar de ello, ha sido capaz de establecer la exigencia de competir como regla del juego dominante.

La actividad investigadora es concebida como un proceso contínuo de valorización de sí que requiere planificación y aplicación de principios empresariales: producción, inversión, cálculo de costes. La forma reflexiva, la forma que tenemos de relacionarnos con nosotros mismos, toma la apariencia de un conjunto de elecciones racionales basadas en el cálculo económico coste-beneficio y que requieren de autocontrol y autoevaluación continúa y voluntaria (Reyes, 2012). La economía se convierte en una disciplina personal (Laval \& Dardot, 2010/2013).

Decíamos al inicio que la pregunta por las condiciones de posibilidad de la investigación crítica no puede hacerse sin atender a la particular posición de sujeto que los modos de gobierno neoliberales 
producen. Pues bien, icómo pensar desde esta configuración subjetiva las condiciones de posibilidad de una investigación crítica?, ihasta qué punto el contenido de nuestras investigaciones queda relegado a un segundo plano cuando lo que interesa es la valorización de sí?, ien qué medida la individualización del conocimiento nos aísla y separa de los problemas que abordamos?, iel conocimiento es un bien común que debe circular para producir efectos o es una mercancía que responde a la lógica del beneficio?

A partir de estas cuestiones, consideramos políticamente relevante, intentar analizar genealógicamente esta configuración identitaria, considerando el neoliberalismo como modo de gubernamentalidad que interviene tanto en el ámbito institucional como subjetivo. Analizar esa forma singular, histórica de relacionarse con el conocimiento y con nosotros mismos, preguntándonos icómo hemos llegado a ser eso que somos?, ide qué forma somos gobernados?, ¿en nombre de qué principios?, ipara conseguir qué objetivos?, ipor medio de qué procedimientos? Ello nos permitirá, describir y explorar las sujeciones que imposibilitan una producción de conocimientos críticos, así como contribuir a desnaturalizar su carácter de evidencia.

\section{El dispositivo neoliberal: una genealogía}

La forma hegemónica de relacionarse con el conocimiento que intentamos problematizar donde este se aleja de su dimensión social, responde a un conjunto de discursos y prácticas, que utilizando herramientas foucaultianas, denominaremos tecnologías y que modifican la subjetividad en relación con determinados objetivos políticos (Foucault, 1994a, 1978b, 1981). Desde la perspectiva de la gubernamentalidad, a través de dispositivos compuestos de elementos heterogéneos (vocabularios, objetos, prácticas, sistemas de juicio, espacios,...) que construyen nuestra interioridad se persigue un objetivo más o menos consciente de regulación social. La potencia del dispositivo o tecnología reside precisamente en el ensamblaje o agenciamiento de sus elementos que, de forma conjunta, son capaces de producir efectos de gobierno de la subjetividad.
No obstante, si bien consideramos que las tecnologías de gobierno neoliberales pueden definir el sentido de las transformaciones de la universidad, a escala global, reconocemos también que al igual que otros ámbitos sociales o geopolíticos, la universidad no es un conjunto coherente, sino una multiplicidad en la que encontramos transición, crisis y combinaciones locales de discursos y prácticas diversos y contradictorios y que, por ello, alberga distintas tensiones y conflictos y formas de resistencia. Tensiones entre distintas racionalidades políticas emergentes o neoliberales y decadentes o welfaristas, pero también tensiones entre posiciones que disputan la función del conocimiento presentes en múltiples intersticios donde se desarrollan formas de resistencia dentro y fuera del ámbito universitario (Galcerán, 2010; Read, 2010).

Nos proponemos, por tanto, describir los elementos que caracterizan la novedad de las formas de regulación neoliberal que atraviesan la universidad y que han sido capaces de redefinir el valor y uso del conocimiento en la institución universitaria y la interioridad de quienes forman parte de ella en consonancia con lógicas individualizadoras. Para ello, en primer lugar, describiremos la transformación de los discursos que legitiman el trabajo investigador y su papel a la hora de instaurar un sentido común compartido o regla de juego que naturalice la necesidad (individual) de competir. En segundo lugar, mostraremos el potente efecto subjetivador de prácticas como la evaluación o la rendición de cuentas que vinculan la producción investigadora con el reconocimiento (valor, prestigio) diferencial de cada uno/a. En tercer lugar, explicaremos la modificación de los patrones de juicio normativo presentes en la institución universitaria, señalando su capacidad de incitar a la movilización continua y sus efectos prescriptivos sobre la producción reflexiva de conocimiento. En cuarto lugar, insistiremos en el hecho de que estamos ante formas de gobierno toleradas, porque no se experimentan como tales ya que se apoyan en el autocontrol, la libertad e iniciativa de los gobernados.

Con ello, de acuerdo con una aproximación genealógica, intentamos 'convertir en aconteci- 
miento' un modo de subjetividad, es decir, devolverle su carácter singular, histórico, construido, contingente, imprevisible, sin recurrir a esquemas causales o identitarios. Si la evidencia es una forma de invisibilidad que nos hace ver cualquier fenómeno social desconectado de la nube de prácticas históricas a las que debe su emergencia, la genealogía es el instrumento que hace aparecer esas conexiones señalando los procesos múltiples que lo constituyen, definiendo líneas de análisis que lo vuelvan inteligible y que expliquen su naturalización (Foucault, 1980, 1990). Se trata de dilucidar su proceso de constitución, intentando mostrar cómo surge y a qué intereses responde (Ibáñez, 1989); desvelar la heterogeneidad de lo que imaginábamos homogéneo.

\section{La batalla por el sentido: investigadores emprendedores y competitivos}

La lógica neoliberal ha ganado la batalla por el sentido. Ha conseguido consolidar una forma de pensamiento, una manera de plantear los problemas $y$, en consecuencia, un sistema de respuestas que proporciona claves para volver inteligible la realidad de acuerdo a una racionalidad instrumental y competitiva que ha adquirido un carácter aproblemático (Laval \& Dardot, 2010/2013).

Las universidades, con el objetivo declarado de cambio en la cultura del profesorado y con ayuda de los discursos neomanageriales, participan de modo activo en la elaboración de códigos morales que actúan como discursos legitimadores o éticos en la medida en que a través de ellos los distintos colectivos implicados comprenden y juzgan sus prácticas (Fernández, 2007; Zangaro, 2011). Nociones que enfatizan un ideal de autonomía responsable (excelencia, espíritu emprendedor, innovación, autorrealización, creatividad) y que articulan un trabajo pedagógico dirigido a que cada uno se considere poseedor de un capital humano que debe hacer fructificar, maximizando sus intereses, persiguiendo lógicas individuales en un contexto de competencia.

Estos nuevos discursos legitimadores extraen su potencia y su carga semántica positiva del hecho de autopresentarse como la alternativa y la superación de la vieja racionalidad política welfarista. Y se sostienen en una tensión que se articula como oposición o necesidad de elegir entre un sistema universitario descalificado por burocrático, rígido, homogeneizador frente a una universidad supuestamente "modernizada", flexible, innovadora y competitiva (Du Gay, 2000/2012). Forman parte, por tanto, de la espectacular reconversión del sistema de convenciones, justificaciones y metáforas asociado al enfrentamiento entre formas de organización y regulación emergentes y decadentes. Y constituyen un conjunto de creencias que Boltanski y Chiapello (1999) denominan "el tercer espíritu del capitalismo", representaciones susceptibles de guiar la acción y justificaciones compartidas que hacen del capitalismo -en su versión neoliberalun orden aceptable e incluso deseable.

De este modo, los nuevos discursos que valoran el riesgo inherente a la existencia individual y colectiva y apelan a la capacidad de los sujetos para hacer elecciones y para alcanzar resultados se apoyan en la convicción (y la recrean) de que, al igual que sucede en otros espacios, la protección de derechos o regulaciones externas y colectivas son dañinos para la creatividad, la innovación, la empleabilidad y son interpretadas como corporativismo o falta de compromiso. La crítica a la burocracia disfuncional actúa como justificación de la desregulación 5 . En esta batalla simbólica, frente a las inercias burocráticas se defiende una ética emprendedora, así como la necesidad de flexibilidad y continuo crecimiento (Terrén, 1999).

Se trata de discursos normativos que, al presentarse en nombre de la modernización y racio-

5 No es difícil observar cómo la obsesión neoliberal por la desregulación laboral se abre camino en la universidad a través de discursos compartidos incluso por aquellos/as que sufren la exigencia de competición continua en un mercado laboral desregulado, exigencia que se traduce en incertidumbre y precariedad. Véase, en este sentido, el Informe de la Comisión de Expertos Internacionales de la Estrategia Universidad 2015: "Audacia para llegar lejos: universidades fuertes para la España del mañana” y el Informe de la Comisión de Expertos para la Reforma del Sistema Universitario Español: "Propuestas para la reforma y mejora de la calidad y eficiencia del sistema universitario español". 
nalización, han conseguido, por un lado, desideologizar las políticas que proponen, hacerlas aparecer como neutrales hasta el punto de imponerse como evidencia sobre la que no cabe debatir, solo adaptarse; y por otro, ocupar estratégicamente el espacio de la crítica ya que incorpora, transformando su sentido, algunas de las reivindicaciones más atractivas (innovación, creatividad) que surgieron frente a la lógica disciplinaria, frente a sus límites y deficiencias (Boltanski \& Chiapello, 1999). En este sentido, llevan a cabo una inversión retórica y caricaturesca de los sentidos originarios, basada en una reinterpretación individual de problemas o cuestiones sociales (Crespo \& Serrano, 2013).

De este modo, se consolida un imaginario que privilegia la categoría de investigador/a que publica y obtiene recursos para la institución, cuya responsabilidad se define en consonancia con la necesidad de la universidad (y de uno mismo/a) de competir y no en términos de implicación o compromiso con lo que queda fuera de estas lógicas productivistas. Sujetos activos, al acecho de las mejores oportunidades, incitados siempre a maximizar su interés propio, actores de sus elecciones, capaces de asumir riesgos. Un imaginario que, apelando al deseo de competitividad y al ideal de excelencia, dificulta cualquier crítica que implicaría situarse en el lugar de los perdedores (Montenegro \& Pujol, 2013).

\section{Técnicas evaluadoras: inversión,} imagen, reconocimiento

Sin embargo, consideramos que el proceso de reconfiguración subjetiva que tratamos de describir no responde únicamente a un proceso de cambio ideológico que trataría de influir en los valores y creencias del profesorado. Supone, al mismo tiempo, un proceso que a través de procedimientos prácticos afecta también a los deseos, aspiraciones, motivaciones, placeres. Así, la pluralidad de técnicas basadas en la evaluación y la rendición de cuentas establecen ejercicios de autoformación que producen un sí mismo en el proceso de practicarlo y permiten actualizar una autonomía responsable
(Rose, 1989, 1996b) ${ }^{6}$. Sin ellas, los discursos que hemos señalado en el apartado anterior carecerían de fuerza.

Las distintas prácticas evaluadoras permiten a los individuos gobernarse bajo la presión de la competición. La exigencia de mostrar lo que se hace y someterlo a juicio, valorizando así su producción intelectual, estimula y concentra los esfuerzos de gran parte del profesorado. El rendimiento de cada uno/a es visibilizado y evaluado, de forma continua, como producto medible en un escenario jerarquizador, donde lo que se pone en juego es la propia imagen. Una competición generalizada dentro de los departamentos, entre departamentos, centros, institutos, universidades donde los productos que se derivan de la actividad investigadora son un resultado más que poder exhibir y con el que contar. Los diversos procedimientos para visibilizar logros reflejan la distancia entre lo que uno es y en lo que se puede convertir. Situación que incentiva, más que cualquier discurso o exhortación, una competición continua. Rankings, estadísticas, memorias de investigación, tablas comparativas, informes, plataformas o registros virtuales (de citas, publicaciones, seguidores...) permiten reconocerse y ser reconocidos, darse valor y ser valorados de acuerdo con ella. De este modo, dispositivos de evaluación y mercado e incentivaciones narcisistas se conjugan para conseguir que los individuos intensifiquen su rendimiento, sometidos a exigencias de resultados cada vez más elevados. Lo que se traduce en un aumento de la presión y del estrés laboral, del ritmo de trabajo y una modificación de las relaciones sociales (Ball \& Youdell, 2007).

La individualización de los objetivos, de las recompensas y sanciones, basándose en evaluaciones cuantitativas impulsa un trabajo ético que debe actualizar la agencia de cada uno. De este modo, al presentar el éxito o fracaso como mero resultado de elecciones personales, se refuerza una concepción

6 Considerar las prácticas evaluadoras de la producción científica como tecnologías de la subjetividad desplaza la reflexión sobre la objetividad o adecuación de los criterios utilizados en la evaluación hacia la construcción del sujeto que efectúan (Amigot \& Martínez, 2013, 2015; Ema, 2013; Gómez \& Jódar, 2013). 
utilitaria del conocimiento ligada a la lógica del beneficio (Laval \& Dardot, 2010/2013).

La práctica investigadora es transformada en una inversión en el propio currículo que reporta al investigador/a beneficios subjetivos y/o materiales al tiempo que dificulta procesos de pensamiento y reflexión colectivos, que cuando se dan, corren el riesgo de ser capitalizados por la lógica de la autoría individual. De forma que la producción de conocimiento, al margen de las posiciones teóricas y políticas de los investigadores/as, se encierra en un circuito privatizado, ajeno a cualquier compromiso con lo común. Al mismo tiempo, se asume que no responder de modo continuo a determinados estándares (que se elevan progresivamente) supone una amenaza constante de posibles pérdidas (según la posición que se ocupe), de la que además cada uno/a será responsable. Se produce una interiorización de las contradicciones que invisibiliza el conflicto. La crítica se transforma en psicología, en prescripción individualista de cambio subjetivo (Castro, 2010; Gómez, Jódar, \& Martínez, 2006). El management neoliberal priva al trabajo investigador de su horizonte común, fragmentando y segregando a los individuos, impidiendo la acción colectiva (Laval \& Dardot, 2010/2013).

\section{Una movilización permanente: \\ hiperactividad y dependencia}

Los nuevos sentidos y las prácticas evaluadoras se conjugan, como acabamos de ver, para incentivar una activación continua del capital humano tanto personal como académico de acuerdo con criterios productivistas. Para entender mejor este proceso y la potencia del dispositivo que estamos describiendo, hay que introducir un tercer elemento que nos hace entender la singularidad de los modos de gobierno neoliberales: la renuncia a establecer patrones normativos con límites claramente establecidos y que, por ello, adquieran una apariencia coercitiva.

La norma con su connotación de estado permanente, de pauta claramente delineada a la que los sujetos deben adaptarse -característica de una lógica disciplinaria- pierde sus contornos y por tanto su visibilidad, se transforma progresivamen- te en una demanda sin límites nítidos, borrosa, desdibujada, flexible, heterogénea. Una demanda que apela a la capacidad constante de avanzar más, de explotar al máximo las propias potencialidades (Comisión Europea, 2005). Como advertía Deleuze (1990/1996) en las sociedades de control, nunca se termina nada, nos convertimos en sujetos en deuda.

La universidad-empresa desplaza la norma disciplinaria entendida como molde por controles que permiten una modulación continua y variable. El objetivo no es solo la adaptación y la integración, sino la intensificación de los rendimientos en un campo de competición (Laval \& Dardot, 2010/2013). Sin embargo, a pesar de ello, sigue vigente un patrón de juicio normativo en el que los criterios de adaptación son más exigentes. La promesa de reconocimiento, de valoración, es siempre conducida por medio de operaciones que distinguen al mismo tiempo que identifican. Los diferentes dispositivos de evaluación de la producción investigadora se basan no sobre procesos rígidos de inclusión/exclusión, sino sobre procesos de inclusión diferencial (Colectivo Edu-Factory, 2010) ${ }^{7}$. Una inclusión precaria que confronta logros individuales tiene que ponerse a prueba constantemente. Este proceso permite justificar la exigencia de sobrecargas laborales y se traduce en una extensión del trabajo y de la responsabilidad personal hacia límites poco definidos. La frontera entre el tiempo de trabajo y los otros tiempos se diluye (cuidados, crianza, relaciones no laborales, participación política...).

La producción de conocimiento se valora de acuerdo a un horizonte inalcanzable que supone hiperactividad y esfuerzos constantes, ninguno de ellos completamente satisfactorio ni definitivo, pero que, sin embargo, obliga a estar siempre en movimiento, permanentemente en curso y a demostrar la flexibilidad necesaria para digerir cantidades cada vez mayores de exigencias. Ya no hay logros acabados y duraderos, no hay metas fijas a las que

7 Sin embargo, no todo el colectivo tiene las mismas posibilidades de alcanzar los resultados que se presentan como potencialmente alcanzables por todas/os, sino que depende de su posición diferencial en el contexto académico-laboral: la posición de género y las redes sociales en las que cada uno/a está inserto continúan distribuyendo desigualmente a los incluidos y excluidos. 
se pueda llegar ni tampoco tiempos vacíos en la carrera investigadora. Estas exigencias ponen en peligro el carácter artesanal, reflexivo y creativo de los procesos de producción de conocimiento: la elección de temas se somete a la lógica de la oportunidad, respondiendo a lo que demanda la investigación financiada y el conocimiento elaborado se transforma en producto que pueda ser aceptado por aquellas revistas que certifiquen que tienen calidad (ajustando y modificando para ello ritmos de trabajo, perspectivas teóricas, metodologías, resultados,...). Se abandona progresivamente o se descuida todo aquello que no resulte rentable de acuerdo con estos criterios de valoración: monografías o libros, determinados estilos de pensamiento y escritura, relaciones no útiles o potencialmente instrumentables, incluso la propia tarea docente (devaluada respecto a la tarea investigadora que es la que otorga prestigio).

\section{La paradoja de una sujeción con agencia}

Hemos descrito el funcionamiento de una tecnología que se apoya en la persuasión inherente a sus discursos legitimadores, en la atracción ejercida por el reconocimiento que promete y en la ansiedad que genera someterse a metas imposibles de alcanzar. Y por ello, una tecnología de gobierno que no se percibe como tal. En este punto (final) del recorrido, queremos detenernos en el núcleo de los modos de gubernamentalidad neoliberal (presente también en los distintos ángulos que hemos descrito): los procedimientos de control externo se transforman en mecanismos de autocontrol. El ejercicio del poder apela directamente a la responsabilidad e implicación voluntaria de los individuos (Barry, Osborne, \& Rose, 1996; Burchell, Gordon \& Miller, 1991). El sujeto de gobierno es un sujeto libre y autónomo (Dean, 1999; Rose, 1999).

Ya no se trata de producir sujetos dóciles y disciplinados a través de la coacción, sino de producir una subjetividad que debe estar completamente implicada en la actividad que debe llevar a cabo. Al igual que sucede en otros ámbitos, la lógica neoliberal no pretende anular la iniciativa de los gobernados sino trabajar sobre ella continuamente para utilizarla a su favor (Deleuze, 1996) ${ }^{8}$. La libertad se convierte en una exigencia y en un mandato, se produce, organiza y administra (Castro, 2010). Para ello, se moviliza la voluntad de autorrealización (aspiraciones, motivaciones) de forma que coincida con los intereses de la organización, a través de su identificación con los valores, la visión y la misión que esta defiende (Zangaro, 2011). De forma que la tarea investigadora se re-conceptualiza como imperativo psicológico, como oportunidad de desarrollo de las propias capacidades que promete reconocimiento, valoración y visibilidad. Apelar a la propia motivación resulta más eficaz que la mera obligación externa, porque incorpora un régimen de autodisciplina que instrumentaliza el deseo y la culpa. El esfuerzo se intensifica voluntariamente porque no responde a una demanda exterior sino interior. Las prácticas de autogestión del yo invisibilizan las demandas, el poder se vuelve opaco instalado en cuerpos particulares que son impelidos a asumir que la solución de los conflictos y carencias solo depende de la esfera de su acción individual (Castro, 2010).

De este modo, los sujetos se vinculan a un proyecto de identidad que les obliga a desplegar estrategias para incrementar su capital humano y valorizarlo de la mejor manera posible y que adquiere sentido en la medida en que se interpreta como una elección personal (Laval \& Dardot, 2010/2013). Paradójicamente, la autonomía personal no es la antítesis de la sujeción, sino un elemento fundamental para su ejercicio (Dean, 1999; Rose, 1996, 1999). La regulación neoliberal se apoya en las prácticas voluntarias o tecnologías del sí mismo que permiten a los individuos efectuar un número de operaciones en sus propios cuerpos, en sus pensamientos, en sus conductas con el fin de transformarse a sí

8 La transformación de los mecanismos de gestión de riesgos que habían caracterizado al régimen welfarista exige reemplazar al individuo dependiente y necesitado, al ciudadano social ligado a la colectividad por mecanismos estatales de solidaridad, en un individuo activo y autorresponsable, empresario de sí mismo. En los distintos ámbitos y esferas, los sujetos son permanentemente estimulados a responsabilizarse, a ser activos, ya sea para contratar un servicio de salud, acceder a una vivienda, buscarse un empleo, proveerse de seguridad física, modificar sus hábitos alimentarios o practicar y preservar un determinado estilo de vida (Rose, 1996b). 
mismos en función de determinados valores y criterios (Foucault, 1984b, 1988). De este modo, ética y política se confunden (Vázquez, 2005). En estas racionalidades, que se sostienen en la exigencia de autorresponsabilidad, las tecnologías del sí mismo refuerzan y consolidan las estrategias más generales de gobierno neoliberal. De forma que las prácticas que desplegamos voluntariamente para amplificar las capacidades investigadoras responden a un conjunto de identificaciones -que no cuestionamos o cuestionamos con dificultad- dirigidas a producir comportamientos funcionales a las necesidades de la organización, a maximizar nuestra utilidad. Y que por ello, alcanzan, sin duda, el colmo de la alienación al pretender suprimir todo sentimiento de alienación (Laval \& Dardot, 2010/2013). Identificaciones cuyo denominador común consiste en responder, en todos los casos, a una gramática individualizadora y que además de clausurar la producción investigadora en términos autorreferentes, tienen capacidad de convertir esta forma histórica de relacionarse con el conocimiento en aproblemática, natural, deseable.

\section{Reflexiones finales: privatización del conocimiento y nuevas praxis instituyentes}

La red de prácticas que el análisis genealógico hace aparecer nos permite explicar el proceso de constitución de una forma singular de relacionarnos con el conocimiento y con nosotros mismos: su emergencia, contingencia y vulnerabilidad. El análisis muestra que nuestra interioridad está configurada por discursos legitimadores o éticos (excelencia, emprendimiento, empleabilidad, innovación); prácticas (evaluación, rendición de cuentas, planificación estratégica); objetos (rankings, memorias, informes); patrones de juicio (optimización continua e ilimitada de nuestro rendimiento); modos de relación con uno mismo (autorregulación, autocontrol); ideales (autorrealización) y cambios legislativos. La subjetivación es un proceso de unificación, de estabilización relativa de todos estos elementos heterogéneos (elementos que aisladamente carecerían de efectos de regulación de las conductas) siempre relativa a un determinado momento histórico y que construye nuestra experiencia (Rose, 1996b).

Un proceso que nos ha convertido en investigadores competitivos que persiguen la superación indefinida de uno mismo, pero siempre en relación con exigencias o deseos que ponen en juego el propio éxito o el fracaso y que, por ello, nos permite entender el proceso por el que la producción de conocimiento pierde su dimensión social. En este punto, retomando la pregunta inicial: ise puede producir un saber crítico cuando la misma producción de conocimiento se ha convertido en un modo de gobierno neoliberal de la subjetividad?, consideramos que el modo de relacionarnos con el conocimiento que estamos describiendo (individualizado, privatizado, estandarizado, autorreferente) supone un obstáculo para el pensamiento crítico porque convierte la implicación del conocimiento con determinadas realidades y problemas sociales en una apuesta continua de valorización de sí (reconocimiento, autorrealización) en un circuito de competencia. Es decir, configura una posición de des responsabilidad, indiferencia y separación frente a lo que queda fuera del interés personal. La realidad se convierte en un tema sobre el que indagar y permanece separada, ajena a las propias afecciones, es un objeto que no compromete (Garcés, 2013). Este modo de relacionarse con el conocimiento consolida el carácter naturalizado de la realidad social y en él, se encuentra el germen de la impotencia política, uno de los efectos de la subjetivación neoliberal'.

Del mismo modo, la búsqueda de reconocimiento a través de la difusión del conocimiento en circuitos cerrados y sostenida en logros individualizadores (autoría individual, copyright, patentes), dificulta su valor como bien común, tanto en lo que hace referencia a su operatividad real, a su conexión con determinadas prácticas, posiciones, problemas, a su capacidad de intervenir en lo social, como a

9 La subordinación a las lógicas productivistas e individualizadoras que estamos describiendo es congruente, no sin tensiones y conflictos, con la desactivación de la función crítica de la universidad, de su compromiso con la transformación de la sociedad de la que forma parte. Y solo por medio de la crítica, la institución puede albergar la esperanza de "incidir en algo real" y "permanecer en contacto con el mundo" (Boltanski, 2014, p. 246). 
la posibilidad de construir formas participativas de pensamiento e investigación. En este sentido, observamos que es posible, en el circuito prescrito de producción y difusión del conocimiento, la incorporación de autores/as, temáticas, perspectivas teóricas, opciones metodológicas no hegemónicas, pero, sin embargo, su capacidad antagonista se desactiva o neutraliza bajo la forma de un producto/ mercancía.

Por ello, consideramos que la posibilidad de construir conocimientos y prácticas críticos exige, como punto de partida, la crítica de esta configuración subjetiva. Una crítica que permita desujetarnos de ella. Y ello porque la genealogía además de cumplir una función de diagnóstico de lo que somos hoy, también puede ser utilizada como táctica que nos muestra las líneas de fragilidad y precariedad de los objetos que hoy son evidentes (Foucault, 1983). De este modo, el recorrido que hemos realizado a lo largo del trabajo, nos ayuda a construir algunos interrogantes que cuestionan su carácter de evidencia: ¿Qué efectos producen discursos como el emprendimiento y la innovación en el campo en el que operan?, iEs posible articular otras formas de reconocimiento colectivo a nuestro trabajo que desborden la lógica individualizadora de prácticas evaluadoras?, ¿Es posible aprender a dar valor a vínculos no instrumentales?, ¿No es precisamente la hiperactividad aquello que paraliza los procesos de reflexión y crítica?, ¿Cuál es el precio que hay que pagar por la exigencia de implicación continua e ilimitada?, ¿Y la relación entre desregulación laboral, precariedad y sufrimiento?, ¿Cómo visibilizar sus efectos corrosivos en los otros tiempos: crianza, cuidados, participación política?, ¿Cómo hacer circular nuestras investigaciones por fuera de los circuitos rentables?, ¿De qué manera construir espacios comunes, acciones colectivas en un espacio hiperfragmentado?, ¿A qué intereses responden las identificaciones que se ponen en juego en nuestras aspiraciones, motivaciones, deseos?, ¿Cómo liberar la energía y el esfuerzo concentrado en proyectos de identidad que nos aíslan y fragmentan, que impiden el compromiso?

Sin embargo, el cuestionamiento de la reapropiación neoliberal del conocimiento, requiere tam- bién la capacidad de producir colectivamente nuevas reglas, nuevas praxis instituyentes (en plural) que materialicen otra racionalidad y generen nuevas subjetivaciones políticas. No solo nuevos saberes críticos, sino nuevas prácticas que se pregunten "cómo no ser gobernado de esa forma, en nombre de estos principios, en vista de tales objetivos y por medio de tales procedimientos, no de esa forma, no para eso, no por ellos" (Foucault, 1990/1995, p. 7) y que perfilen contraconductas que permitan materializar un doble rechazo a la gubernamentalidad neoliberal: la negativa a conducirse a sí mismo como una empresa de sí y la negativa a actuar de acuerdo con la norma de competencia (Laval \& Dardot, 2010/2013). En esta línea, y para concluir, apuntamos brevemente, de forma exploratoria, tentativa y parcial, algunas reflexiones derivadas de los interrogantes abiertos en nuestro análisis genealógico. Propuestas dirigidas a instituir lo común (Laval \& Dardot, 2014/2015), en términos de implicación y participación ${ }^{10}$, como principio alternativo a las lógicas de gobierno neoliberales orientadas a:

(i) Producir colectivamente otros sentidos que quiebren el consenso definido por conceptos como: excelencia, emprendimiento, calidad, audacia... mostrando sus efectos individualizadores en el espacio en el que operan (a pesar del atractivo con el que se autopresentan) y generando espacios de disenso dentro de las universidades. Excelencia, como incentivo a un aumento constante e ilimitado de la productividad; calidad, como sometimiento a estándares y formatos cuantitativos y arbitrarios; emprendimiento, como espejismo de agencia y logro personal, en el trabajo investigador que invisibiliza las marcas de género, clase y la pertenencia a redes institucionales; audacia y riesgo como prescripciones que convierten en deseables situaciones de precariedad. Se trataría de romper el efecto

10 Entendemos lo común, siguiendo a Laval y Dardot (2014/2015), como fórmula que emerge de los movimientos de contestación y las corrientes de pensamiento que luchan contra la apropiación privada de todas las esferas de la vida operada por el neoliberalismo. Lo común no es un objeto, tampoco una característica de ciertos tipos de "bienes", sino que es algo que debe ser instituido a través de praxis basadas en la implicación y la deliberación colectiva. Por lo tanto, el valor del conocimiento como 'bien común' es siempre resultado de una praxis instituyente. 
de coherencia, clausura y necesidad producido por el tejido semántico desplegado por la razón neoliberal (Boltanski, 2014), al mismo tiempo que se incorporan otros elementos de valor al trabajo investigador: trabajo compartido, trabajo artesanal, honestidad, compromiso, valor de los vínculos no instrumentales ${ }^{11}$.

(ii) Oponerse a las formas actuales de evaluación de la actividad investigadora en lo que hace referencia a las prácticas que convierten la competitividad el elemento central de la producción de conocimiento (índices de producción científica, rankings, medidas legislativas que sancionan la fragmentación y jerarquización de los colectivos). Para ello, es prioritario que la práctica investigadora pueda salir del circuito de producción y difusión autorreferente que funciona sin exterior, ajeno a las tensiones, malestares y conflictos que atraviesan la sociedad de la que forma parte (Boltanski, 2014). Ello supondría alterar el sentido que se otorga a la rendición de cuentas en relación con la producción de conocimiento no como un valor de cambio ligado a las exigencias del mercado, sino como valor de uso ligado a favorecer su circulación y conexión con determinadas posiciones y problemas, articulando prácticas que, desbordando la lógica de la autoría individual y del paper de investigación científica como formato privilegiado, permitieran formas abiertas y colectivas de construcción y difusión del conocimiento producido (prácticas de autoría colectiva, espacios o foros abiertos de discusión y difusión no académicos...). En este sentido, las experiencias de autoformación a las que nos hemos referido pueden servir como referente porque constituyen un laboratorio de prácticas institucionales innovadoras basadas en presupuestos democráticos de horizontalidad, colaboración e independencia dirigidas a mostrar tanto la capacidad transformadora de los procesos de reflexión compartida ${ }^{12}$

11 La "Carta de la Desexcelencia" (Enero de 2014) impulsada por un grupo de universidades francesas y belgas o el movimiento Slow Science (2010) constituyen ejemplos de aperturas politizadoras.

12 Frente a dispositivos de conocimiento desencarnados, circulares e impotentes, estas experiencias alternativas de autoformación remarcan el vínculo entre las mutaciones perceptivas y afectivas que se derivan de la producción colectiva de saberes centrados como la necesidad (situada, estratégica) de llevar a cabo investigaciones que se enfrenten al reto de construir lo común en un espacio hiperfragmentado. Prácticas que entienden la investigación crítica como articulación con posiciones y colectivos que forman parte de las luchas por la definición de lo social (Fractalitats en Investigació Crítica, 2005).

(iii) Cuestionar aquellas políticas laborales que promueven en el personal docente e investigador situaciones de desregulación laboral y precarización, porque impiden que las posiciones más vulnerables del sistema universitario (jóvenes investigadores, profesorado no estable) generen formas de resistencia a este modo privatizado de relacionarse con el conocimiento. Estas posiciones se convierten en engranaje obligatorio de la maquinaria productivista y competitiva, porque de la consecución de estándares que se elevan progresivamente depende el valor que se otorgue a su competencia profesional (su nivel de reconocimiento), pero sobre todo su potencial empleabilidad, esto es, la posibilidad mantener (o de conseguir en un horizonte incierto) el propio puesto de trabajo.

De ahí que se vuelve necesario visibilizar las condiciones materiales (exigencias de flexibilidad temporal, funcional y horaria, inestabilidad laboral...) y subjetivas (ansiedad, desgaste psíquico, incertidumbre, culpa) que definen hoy la producción de conocimiento en la universidad. Situaciones de fragilidad subjetiva (Amigot \& Martínez, 2013, 2015) que generan dependencia hacia criterios de valoración que promueven determinados estilos de pensamiento y escritura, que prescriben parámetros temporales, contenidos prioritarios ${ }^{13}$, metodologías legítimas, modos de enunciación y disciplinan, estandarizan y empobrecen el trabajo investigador. Visibilización que requiere lo que Boltanski (2014) denomina crítica radical o existencial centrada en dar legitimidad y volver comprensibles experiencias descalificadas o silenciadas como "subjetivas"14,

en cuestiones que importan y que por ello, tienen efectos politizadores en las propias vidas (Universidad Nómada, 2008).

13 Acordes, en muchos casos, con las actuales formas de producción y con el pensamiento hegemónico dominante (Fractalitats en Investigació Crítica, 2005).

14 Estas experiencias han sido difíciles de tematizar debido a las 
permitiendo pasar de la acumulación de paradojas y contradicciones vividas de modo individual a sostener una posición colectiva que reclame cambios institucionales que cuestionen la política del conocimiento neoliberal y sus efectos (Amigot \& Martínez, 2015).

(iv) Favorecer la constante deliberación y discusión de las reglas institucionales que definen el modo en que nos relacionamos con el conocimiento para evitar su naturalización burocrática. Ello requiere la impugnación de mecanismos de (pseudo) participación neomanagerial incorporados en los últimos años a diversos ámbitos de la gestión universitaria (planificación estratégica, matriz DAFO). Procedimientos de carácter tecnocrático que aplican el esquema problema-solución y permiten una participación limitada al marco de condiciones ya dado, convirtiéndose, por ello, en herramientas de producción de consenso. Rechazan lo que no puede ser codificado, regulado, ordenado, calculado de acuerdo a las coordenadas previas. Frente a ellos, sería necesario habilitar prácticas colectivas que no estuvieran dirigidas a la búsqueda de una solución en un marco de condiciones ya dado, tampoco a conseguir que determinadas opciones políticas pudieran expresarse, sino que pusieran en juego la misma posibilidad de política, pensamiento y subjetivación (Ema, 2013), entendida como gesto o posición subjetiva dirigida a la apertura de preguntas que redefinan e impugnen el mismo marco, que cuestionen y transformen los sentidos hegemónicos, posición capaz de quebrar identificaciones asignadas por el orden establecido y de asumir responsabilidades para la vida en común.

reglas del juego de la lógica neoliberal que provocan, como hemos mostrado, una privatización del conflicto. Sin embargo, en los últimos años, determinadas movilizaciones por parte de colectivos de investigadores precarios muestran los efectos corrosivos de sus condiciones laborales (trabajo flexible, inestable que dificulta la construcción de lazos, pone en peligro la continuidad de proyectos vitales y colectivos). Un ejemplo de esta crítica basada en "pruebas existenciales" lo constituye el proyecto dirigido a visibilizar las consecuencias de la movilidad trasnacional "Folow the Felow" (https://followthefellow.wordpress.com/).

\section{Referencias}

Amigot, P., \& Martínez, L. (2013). Gubernamentalidad neoliberal, subjetividad y transformación de la universidad. La evaluación del profesorado como técnica de normalización. Athenea Digital, 13(1), 99-120.

Amigot, P., \& Martínez, L. (2015). Procesos de subjetivación en el contexto neoliberal. El caso de la evaluación del profesorado y la investigación universitaria. Revista de la Asociación de Sociología de la Educación, 8(2), 138-155.

Ball, S., \& Youdell, D. (julio, 2007). Privatización encubierta de la educación pública. Informe preliminar presentado en el V Congreso Mundial de la Internacional de la Educación, Berlín, Alemania. Recuperado de http://www.ei-ie.org/spa/news/ homepage/home

Barry, A., Osborne, T., \& Rose, N. (Eds.). (1996). Foucault and political reason. Liberalism, neoliberalism and rationalities of governement. Londres: UCL Press.

Boltanski, L. (2014). De la crítica. Compendio de sociología de la emancipación. Madrid: Akal.

Boltanski, L., \& Chiapello, E. (1999). Le nouvel esprit du capitalisme. París: Gallimard.

Burchell, G. (1996). Liberal government and the techniques of the self. En A. Barry, T. Osborne \& N. Rose (Eds.), Foucault and political reason. Liberalism, neoliberalism and rationalities of government (pp. 19-36). Londres: UCL Press.

Burchell, G., Gordon, C., \& Miller, P. (Eds.). (1991). The Foucault effect. Studies in governmentality. Hemel Hempstead, UK: Harvester Wheatshef.

Castro, R. (2010). Neoliberalismo y gobierno de la vida. En S. Arribas, G. Cano \& J. Ugarte (Coords.), Hacer vivir, dejar morir. Biopolítica y capitalismo (pp. 63 89). Madrid: CSIS.

Colectivo Edu-Factory. (2010). Nosotros no pagaremos vuestra crisis. iTodo el poder a la autoformación! En Edu-Factory \& Universidad Nómada (Comps.), La universidad en conflicto. Capturas y fugas en el mercado del saber (pp. 41-49). Madrid: Traficantes de Sueños.

Collectif de chercheurs de l'Université libre de Bruxelles. (Enero de 2014). La charte de la désexcellence. Re- 
cuperado de: http://lac.ulb.ac.be/LAC/charte_files/ Charte_Desexcellence_1-1.pdf

Comisión de Expertos Internacionales de la Estrategia Universidad 2015. (2011). Audacia para llegar lejos: universidades fuertes para la España del mañana (Informe final). Recuperado de http://www.usc. es/export/sites/default/gl/web/descargar/informe-finaleu2015.pdf

Comisión de Expertos para la Reforma del Sistema Universitario Español. (2013). Propuestas para la reforma y mejora de la calidad y eficiencia del sistema universitario español (Informe). Recuperado de http://www.mecd.gob.es/dms/mecd/ministerio-mecd/servicios-al-ciudadano/participacion-publica/sistemauniversitario/propuestas-reforma.pdf

Comisión Europea. (2003). Comunicación de la comisión. El papel de las universidades en la Europa del conocimiento (COM 58 de 05.02.2003). Recuperado de http://www.mecd.gob.es/dctm/boloniaeees/documentos/02que/universidades-europaconocimiento. pdf?documentId=0901e72b8004aa8d

Comisión Europea. (2005). Comunicación de la Comisión. Movilizar el capital intelectual de Europa: crear las condiciones necesarias para que las Universidades puedan contribuir plenamente a la estrategia de Lisboa. COM 152 de 20.04.2005. Extraído el 8 de febrero de 2012 de http://www.crue.org/export/sites/ Crue/procbolonia/documentos/antecedentes/7._ El_papel_de_las_universidades.pdf

Comisión Europea. (2006). Comunicación de la Comisión al Consejo y al Parlamento Europeo. $\mathrm{Cu}$ mplir la agenda de modernización para las universidades: educación, investigación e innovación (COM 208 de 10.05.2006). Recuperado de http:// eur-lex.europa.eu/LexUriServ/LexUriServ.do?uri=COM:2006:0208:FIN:es:PDF

Comisión Europea. (2008). La iniciativa emprendedora en la enseñanza superior, especialmente en estudios no empresariales (Resumen del Informe final del grupo de expertos). Recuperado de http://www. cdiex.org/aprendizaje/documento_8.pdf

Crespo, E., \& Serrano, A. (2013). Las paradojas de las políticas de empleo europeas: de la justicia a la terapia. Universitas Psychologica, 12(4), 1111-1124.

Dardot, P., \& Laval, C. (2014). Commun. Essai sur la révolution au XXI siècle. París: La Découverte.
Dean, M. (1999). Governmentality. Power and rule in modern society. Londres: Sage.

Deleuze, G. (1996). Post-scriptum sobre las sociedades de control. En Conversaciones (pp. 277-286). Valencia: Pre-Textos.

Du Gay, P. 2012). En elogio de la burocracia. Weber. Organización. Ética (J. Fernández, Trad.). Madrid: Siglo XXI.

Ema López, J. E. (2013). Límites y oportunidades de lo político en la universidad. La evaluación y sus tropiezos. Athenea Digital, 13(1), 59-79.

Fernández Rodríguez, C. J. (2007). El discurso del management: tiempo y narración. Madrid: CIS.

Foucault, M. (1994a). La philosophie analytique de la politique. En M. Foucault, Dits et écrits (Vol. 3, pp. 534-551). París: Gallimard.

Foucault, M. (1994b) La gouvernementalité. En M. Foucault, Dits et écrits (Vol. 3, pp. 635-656). París: Gallimard.

Foucault, M. (1994c). Table ronde du 20 mai 1978. En M. Foucault, Dits et écrits (Vol. 4, pp. 20-34). París: Gallimard.

Foucault, M. (1994d). “Omnes et singulatim”: vers une critique de la raison politique. En M. Foucault, Dits et écrits (Vol. 4, pp. 134-161). París: Gallimard.

Foucault, M. (1994e). Structuralisme et poststructuralisme. En M. Foucault, Dits et écrits (Vol. 4, pp. 431-457). París: Gallimard.

Foucault, M. (1994f). Foucault. En M. Foucault, Dits et écrits (Vol. 4, pp. 631-636). París: Gallimard.

Foucault, M. (1994g). Une esthétique de l'existence. En M. Foucault, Dits et écrits (Vol. 4, pp. 730-735). París: Gallimard.

Foucault, M. (1994h). Les techniques de soi. En M. Foucault, Dits et écrits (Vol. 4, pp. 783-813). París: Gallimard.

Foucault, M. (1995). ¿Qué es la crítica? Daimon. Revista de Filosofía, 11, 5-25.

Foucault, Michel. (1977). Entretien avec Michel Foucault. En Foucault (1994), Dits et écrits (III, 140. 160) Paris: Gallimard.

Fractalitats en Investigació Crítica. (2005). Investigación crítica: desafíos y posibilidades. Athenea Digital, 8, 129-144.

Galcerán, M. (2010). La educación universitaria en el centro del conflicto. En Edu-Factory \& Universi- 
dad Nómada (Comps.), La universidad en conflicto. Capturas y fugas en el mercado del saber (pp. 13-39). Madrid : Traficantes de Sueños.

Garcés, M. (2010). Dar que pensar. Sobre la necesidad política de nuevos espacios de aprendizaje. En Espai en Blanc. El combate del pensamiento (pp. 67-79). Barcelona: Bellaterra.

Garcés, M. (2013). Un mundo común. Barcelona: Bellaterra.

Gómez, L., \& Jódar, F. (2013). Ética y política en la universidad española: la evaluación de la investigación como tecnología de la subjetividad. Athenea Digital, 13(1), 81-98.

Gómez, L., Jódar, F., \& Martínez, L. M. (2006). Psicología, identidad y política en las tecnologías de gobierno neoliberales. Psicología $\mathbb{E}$ Sociedade, 18(1), 7-14.

Herreros, T. (2010). Laboratorios de autoformación, universidades anómalas, nuevas universidades. En Edu-Factory \& Universidad Nómada (Comps.), La universidad en conflicto. Capturas y fugas en el mercado del saber (pp. 13-39). Madrid: Traficantes de Sueños.

Ibáñez, T. (1989). La psicología social como dispositivo deconstruccionista. En T. Ibáñez (Coord.), El conocimiento de la realidad social (pp. 109-133). Barcelona: Sendai.

Laval, C., \& Dardot, P. (2013). La nueva razón del mundo. Ensayo sobre la sociedad neoliberal. Barcelona: Gedisa.

Laval, C., \& Dardot, P. (2015). Común. Barcelona: Gedisa.

Ministerio de Ciencia e Innovación. (2008). Estrategia Universidad 2015: universidades para el progreso, el bienestar y la competitividad. Recuperado de http://www.redtcue.es/biblioteca/ecosistema/5-estrategia-universidad-2015/file

Ministerio de Ciencia e Innovación. (2010). Estrategia Universidad 2015: el camino para la modernización de la Universidad. Recuperado de http://www. studiaxxi.com/site/wp-content/uploads/2010-pdfeu2015.pdf

Montenegro Martínez, M., \& Pujol Tarres, J. (2013). La fábrica de conocimientos: in/corporación del capitalismo cognitivo en el contexto universitario. Athenea Digital, 13(1), 139-154.
Read, J. (2010). La experiencia de la universidad: el neoliberalismo contra los commons. En Edu-Factory \& Universidad Nómada (Comps.), La universidad en conflicto. Capturas y fugas en el mercado del saber (pp. 99-102). Madrid: Traficantes de Sueños.

Reyes Lara, D. (2012). Mentalidades de gobierno, subjetividad y conocimiento académico: nuevas formas de gobierno de la producción de conocimiento académico en la universidad pública española (Tesis doctoral). Universitat Autònoma de Barcelona, España.

Rose, N. (1989). Governing the soul. The shaping of the private self. Londres: Routledge.

Rose, N. (1992). Governing the enterprising self. En P. Heelas \& P. Morris (Eds.), The values of the enterprise culture. The moral debate (pp. 141-164). Londres: Routledge.

Rose, N. (1996a). Power and subjectivity: Critical history and psychology. En C. F. Graumann \& K. J. Gergen (Eds.), Historical dimensions of psychological discourse (pp. 103-124). Nueva York: Cambridge University Press.

Rose, N. (1996b) Inventing ourselves. Psychology, power and personhood. Cambridge: Cambridge University Press.

Rose, N. (1999). Powers of freedom. Reframing political thought. Cambridge: Cambridge University Press.

Rose, N., \& Miller, P. (1992). Political power beyond the state: Problematics of government. British Journal of Sociology, 43(2), 173-205.

Slaughter, S., \& Leslie, L. (1997). Academic capitalism. Baltimore: The Johns Hopkins University Press.

The Slow Science Academy (2010). Slow Science Manifesto. Recuperado de http://slow-science.org/

Universidad Nómada. (mayo, 2008). Prototipos mentales e instituciones monstruo. Algunas notas a modo de introducción. Transversal, 5. Recuperado de http://eipcp.net/transversal/0508

Terrén, E. (1999). Educación y modernidad. Entre la utopía y la burocracia. Barcelona: Anthropos.

Vázquez, F. (2005). Tras la autoestima. Variaciones sobre el yo expresivo en la modernidad tardía. San Sebastián: Garkoa.

Zangaro, M. (2011). Subjetividad y trabajo: el management como dispositivo de gobierno. Trabajo y Sociedad, 15(16), 163-177. 
